\title{
Feline Diabetes Mellitus and Differential Diagnosis We Need to Consider
}

\author{
Madalina ROSCA BURLACU*, Gheorghe SOLCAN \\ Small Animal Internal Medicine Department, University of Agronomic Sciences and Veterinary Medicine \\ of Iasi, Faculty of Veterinary Medicine, 8 Mihail Sadoveanu Alee, 700489, Iasi, Romania \\ *Corresponding author: m.burlacu.usamv@gmail.com
}

Bulletin UASVM Veterinary Medicine 72(2) / 2015,

Print ISSN 1843-5270; Electronic ISSN 1843-5378

DOI:10.15835/buasvmcn-vm: 11329

\begin{abstract}
Feline diabetes mellitus is a complex pathology and new information is being provided in a fast manner by researches. The diagnosis of type II diabetes mellitus is relatively simple and is based on the observation of sustained high blood glucose level, on two or more occasions. Even so, sustained hyperglycaemia can also occur as a consequence, be associated with, or even induce a series of other pathologies.

The current study targets an accurate differential diagnosis of primary feline diabetes mellitus, associated pathologies and other endocrinopathies which hold a strong diabetogenic potential.

A number of 65 cats diagnosed with type II diabetes mellitus have been included for the development of the study. All cats underwent clinical and physical examination, and further paraclinical investigations have been recommended for the diagnosis with the highest probability. Based on a case data set, targeted diagnoses included: a) primary diabetes mellitus, with no other associated pathologies; b) diabetes mellitus induced by (b.1) hypersomathotropism, (b.2) hyperthyroidism, (b.3) hyperadrenocorticism and (b.4) diabetogenic medication; c) diabetes mellitus in association with (c.1) chronic kidney failure and (c.2) heart failure and d) pancreatitis;

Based on the differential diagnosis, the observed diabetic felines were framed in one or more of the following groups: (a) - 27, (b.1) - 2, (b.2) - 1, (b.3) - 0, (b.4) - 38, (c.1) - 18, (c.2) - 10, (d) - 26.

Diabetes mellitus can occur as primary or sole pathology, and also associated with other endocrinopathies or systemic illness. Most cases of secondary diabetes mellitus display difficult control of blood glucose level, highly variable insulin requirements, a low quality of life and a shorter survival rate. Thus accurate diagnosis might be most important aspect for the future outcome of the disease.
\end{abstract}

Keywords: feline diabetes mellitus, hyperadrenocorticism, hyperthyroidism, hypersomathotropism, insulin resistance

\section{INTRODUCTION}

The diagnosis of feline type II diabetes mellitus (DM) is relatively simple and is generally based on the observation of sustained high blood glucose level on two or more occasions (Blois et al., 2010). In primary DM, general biochemistry usually indicates hypercholesterolemia, hyperlipemia, high liver enzymes, increased specific pancreatic lipases, (fPLI - pancreatic lipase immunoreactivity, fTLI - trypsinogen-like immunoreactivity) when associated with pancreatitis, pre-renal azotaemia, hyperbilirubinemia (severe in liver lipidosis) and moderate to severe hypokalemia. Sustained hyperglycaemia and insulin resistant DM can also occur as a consequence, or in association with other systemic illnesses (ScottMoncrieff, 2010). Differential diagnosis panel includes other primary endocrinopathies, such as hypersomathotropism (acromegaly), hyperthyroidism and hyperadrenocorticism (Cushing syndrome), followed by maldigestion and malabsorption syndromes, organ failure (kidney and heart), iatrogenic DM and pancreatitis induced by toxic states, bacterial, viral and/ 
or parasitic diseases (Mooney and Peterson, 2004, Ettinger and Feldman, 2005, Rucinsky et al., 2010). In what concerns endocrine diseases, hypersomathotropism is one of the most important requiring exclusion, especially in cases of insulin resistant DM, as all acromegalic cats confirmed until present were also diabetic. When suspected, diagnosis for acromegaly is established based on high levels of feline specific basal growth hormone (fGH) if available, high insulin like growth factor 1 (IGF-1) and intracranial MRI evidencing tumours, or increased volume of the pituitary gland. Hyperthyroidism can also be associated or induce insulin resistant DM, some studies reporting a rare association of the two, while others report an incidence of co-morbidities of up to 73\% (Blois et al., 2010, Williams et al., 2010). Accurate diagnosis can be achieved by determining basal thyroxine, total free thyroxine and canine specific TSH when test are available (feline specific not yet developed) (Mooney and Peterson, 2012). Diagnosis of hyperthyroidism can be hampered by non-thyroidal illnesses, which can decrease the level of thyroid hormones within normal parameters, leading to the sick euthyroid syndrome (Peterson et al., 2001, Blois et al., 2010). Thyroidal radioisotope uptake (Scott-Moncrieff, 2010) (holds a low worldwide availability for clinicians) is a highly specific diagnosis technique for hyperthyroidism, measuring an increased percentage of technetium-99 or pertechnetate uptakes in affected thyroid lobes. Another important endocrine disease, but occurring rarely in felines, is hyperadrenocorticism. The diagnosis in cats relies mainly on low dose dexamethasone suppression test, followed by urinary cortisol:creatinine ratio and ACTH stimulation test (low specificity) (Mooney and Peterson, 2012). Chronic kidney disease, also need to be excluded as it is often associated with insulin resistance, fluctuating insulin requirements and unstable clinical states of cats. Case monitoring of diabetic felines with chronic kidney disease is even more difficult, as polyuria/polydipsia (PU/ PD) syndrome can persist (Zini et al., 2014). Last, but not least, diabetes mellitus pathophysiology entails a series of blood pressure alterations. Although hypertension and insulin resistance can have a cause-effect relationship, systolic hypertension over $160 \mathrm{mmHg}$ and different states of heart failure, are often observed in insulin resistant individuals (Rand, 2013). Clinical signs of elevated blood pressure are discrete, easy to overlook and if left unattended lead to irreversible organ damage, especially kidney disease.

Most of these diseases, with predilection endocrinopathies, have an insidious evolution, with discrete clinical signs, which are usually common to/or masked by DM and impose great difficulties in controlling blood glucose level (Mooney and Peterson, 2004). The current paper targets an accurate differential diagnosis of primary feline diabetes mellitus, associated pathologies and other endocrinopathies which hold a strong diabetogenic potential, in a well defined population of confirmed diabetic cats.

\section{MATERIALS AND METHODS}

Data was collected from the medical records of cases presented for consultation at the veterinary teaching hospital of Faculty of Veterinary Medicine of Iasi, Romania. Case retrospective registration started with September 2005 and was carried until September 2014. Records of 5175 cats, both male and female, with ages ranging from 4 weeks to 18 years were consulted. Cases were considered eligible for inclusion in the study if they were registered with characteristic clinical signs and a clear diagnosis of DM (Ettinger and Feldman, 2005, Gunn-Moore, 2005). Diagnosis was based on the clinical signs of polyuria-polydipsia, polyphagia associated with weight loss, persistent fasting hyperglycemia over $270 \mathrm{mg} / \mathrm{dl}(80-120 \mathrm{mg} / \mathrm{dl})$, data obtained on the general serum biochemistry and glycosuria (Blois et al., 2010, Reusch, 2011). Data recorded for each diabetic cat included: age, gender, reproductive integrity, weightand breed. All cats underwent clinical and physical examination and further paraclinical investigations have been recommended for the differential diagnosis. Discriminating DM associated pathologies from the entire feline pathology was achieved by choosing the PU/PD syndrome as a starting point, as it is a constant sign of pathological sustained hyperglycemia. Pathologies of interest for the analyzed population included primary diabetes, followed by other endocrinopathies (hypersomatotropism,

hyperthyroidism, hyperadrenocorticism), induced by the administration of medication with diabetogenic potential, diabetes induced by/in association with chronic/acute organ failure (renal/heart) and 
pancreatitis, frequently encountered in bacterial, viral (feline panleukopenia) and parasitic diseases (Haemobartonellosis). Thus, based on a case data set, targeted diagnoses included: a) primary diabetes mellitus, with no other pathologies; b) diabetes mellitus induced by (b.1) hypersomathotropism, (b.2) hyperthyroidism, (b.3) hyperadrenocorticism and (b.4) diabetogenic medication (progestagens and/or glucocorticoids; c) diabetes mellitus in association with (c.1) chronic kidney disease and (c.2) heart failure and d) pancreatitis. Also, the demographics of the studied population have been analyzed. Following the DM diagnosis, additional test were carried out, according to the clinical presentation of each case, in order to detect primary or secondary pathologies thought to interfere with the glucose homeostasis.

\section{RESULTS AND DISCUSSION}

Diabetes mellitus was diagnosed in 65 of the 5175 cats presented during September 2005 to September 2014, revealing a PP of $1.3 \%$ and $95 \%$ CI of $0.99-1.61 \%$. Of the total DM cases, 38 were males $(58.5 \%)$ and 27 females (41.5\%). A number of 13 males (20\%) were intact and 25 (38.4\%) neutered. In the female group, 19 (29.3\%) were intact and $8(12.3 \%)$ neutered. The average age of the group was $10.9 \pm 3.5$ years, with ranges from 3 to 18 years. The mean weight of total 65 DM cases was $4.9 \pm 1.2$ [SD] kg, ranging from 2.8 and $8 \mathrm{~kg}$. The demographics of DM cat population is described in Tab. 1.

A number of 27 cats $(41.5 \%)$ were diagnosed with primary DM, presenting persistent fasting hyperglycemia, glycosuria and progressive weight loss. Also, different degrees of dehydration, ketoacidosis, ketonuria and liver lipidosis have been registered. No other clinical signs have been observed in this group of cases that could indicate the presence of other underlying or pre-existent diseases. All cats responded well to the insulin treatment and none expressed signs of insulin resistance. Acromegaly was diagnosed in two cats of $65(3.07 \%)$, a 14 year old domestic short haired spayed female and a seven year old domestic short haired entire male (Fig. 1). The suspicion of DM secondary to acromegaly was based on the severe insulin resistance, both cases requiring a median dose of $2.1 \mathrm{IU} / \mathrm{Kg} / 12 \mathrm{~h}$ (range: $1.5-3 \mathrm{IU} /$ $\mathrm{Kg} / 12 \mathrm{~h}$ ) of long acting insulin. Also, the physical examination indicated weight gain, in spite of the severe insulin resistance and almost permanent severe hyperglycemia. Other features included broad facial features, widening of the interdental spaces, inferior prognatia, organomegaly (liver and kidneys) and opened mouth breading in the female cat, all characteristics of acromegaly. The diagnosis has been confirmed by determining basal IGF-1 level, with a cut off for diagnosis of $1000 \mathrm{ng} / \mathrm{ml}$ (reference: $200-800 \mathrm{ng} / \mathrm{ml}$ ) for both cats, and intracranial MRI examination, revealing an enlarged pituitary gland in the female cat. Hyperthyroidism was suspected in one cat $(1.5 \%)$ (Fig. 2), which besides the characteristic DM signs, also displayed tachycardia, systolic murmurs, episodes of vomiting and diarrhoea, voracious appetite, severe weight loss, hyperactivity and bilateral palpable goitre. Insulin requirements were fluctuant, with periods of insulin resistance and episodes of severe hypoglycemia, all specific for hyperthyroidism. The diagnosis was confirmed based on elevated levels of total thyroxine 100 nmol/L (range: $15-60 \mathrm{nmol} / \mathrm{L}$ ) and free thyroxine $130 \mathrm{pmol} / \mathrm{L}$ (range: 10-45 pmol/L), bilateral increased volume of the thyroid lobes and observed clinical signs. Synthetic progestagen and/or glucocorticoid associated DM has been identified in 38 cats (58.5\%) (Fig. 3), documented with repeated and/or prolonged administration of these diabetogenic substances. The diagnosis was

Tab. 1. Demographics of DM cat population

\begin{tabular}{ccc}
\hline Group & Diabetic No. & \% of 65 \\
\hline Total & 65 & \\
\hline Gender & & \\
\hline Male & 38 & 20 \\
\hline intact & 13 & 38.46 \\
\hline neutered & 25 & 41.53 \\
\hline Female & 27 & 29.23 \\
\hline intact & 19 & 12.30 \\
\hline neutered & 8 & \\
\hline Age & & 12.30 \\
\hline$<7$ & 8 & 41.53 \\
\hline 7 to 12 & 27 & 46.15 \\
\hline$>12$ & 30 & \\
\hline Weight & & 13.8 \\
\hline$<5 \mathrm{~kg}$ & 22 & 86.2 \\
\hline$>5 \mathrm{~kg}$ & 43 & \\
\hline Breed & & 39.23 \\
\hline Burmese & 32 & 13.84 \\
\hline DSH & 24 & \\
\hline Other & 9 &
\end{tabular}


confirmed by recording a complete history of the cases, acute debut of DM clinical signs and lack of other characteristics. Cats in this category were able to recover from DM and achieve remission in a shorter period of time than other cases. Stages I to IV chronic kidney disease have been diagnosed in 18 cats (27.7\%) (Fig. 4), based on clinical signs, elevated serum creatinine, registering a median of $3.6 \pm 1.4 \mathrm{mg} / \mathrm{dl}$ (reference: $0.5-1.6 \mathrm{mg} / \mathrm{dl}$ ) and urea of $135 \pm 70 \mathrm{mg} / \mathrm{dl}$ (reference: $15-31 \mathrm{mg} /$ dl). Cats with kidney disease proved to be more difficult to monitor, due to persistent sings of $\mathrm{PU} / \mathrm{PD}$, which induced confusions in the insulin dosing regiments. Insulin dosing in this group, also registered frequent fluctuations in the daily requirements. Different degrees of heart disease were diagnosed in 10 cats (15.4\%) based on the hear auscultations and ultrasound examinations. Pancreatitis was suspected in 26 cases (40\%), manifesting clinical signs of decreased appetite to anorexia, moderate to severe dehydration, lethargy, vomiting and diarrhoea episodes and abdominal distension. No cat of the total of 65 displayed characteristic clinical signs of Cushing syndrome, and none of them had been diagnosed with the disease.

The observed diabetic felines were framed in one or more groups, according to the

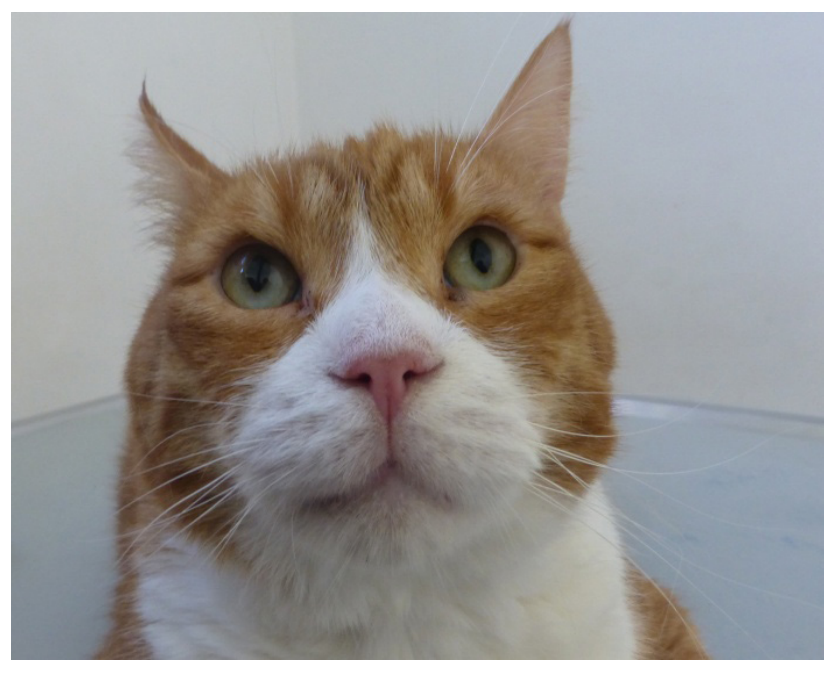

Fig. 1. Insulin resistant DM secondary to acromegaly in an eight year old, DSH male cat;

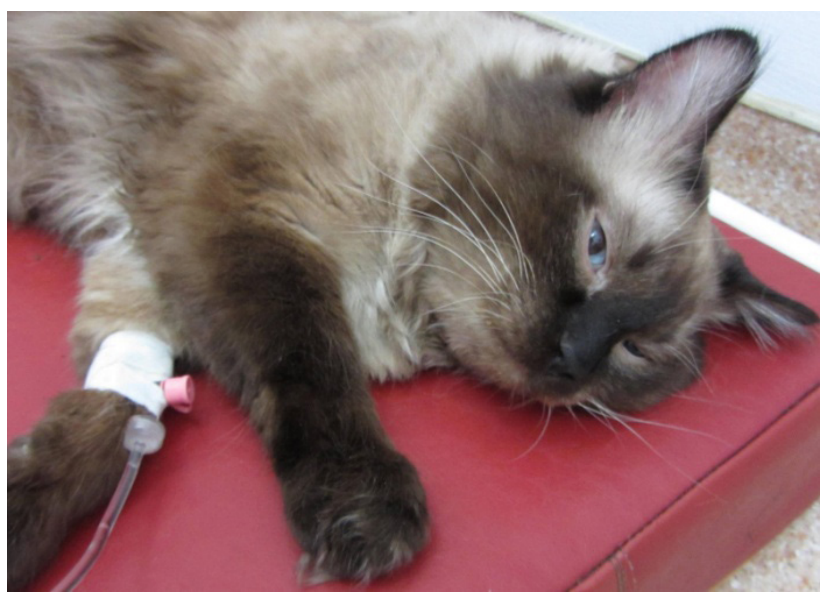

Fig. 3. DM in an 8 year old entire Burmese male cat exposed to high and repeated doses of megestrol acetate;

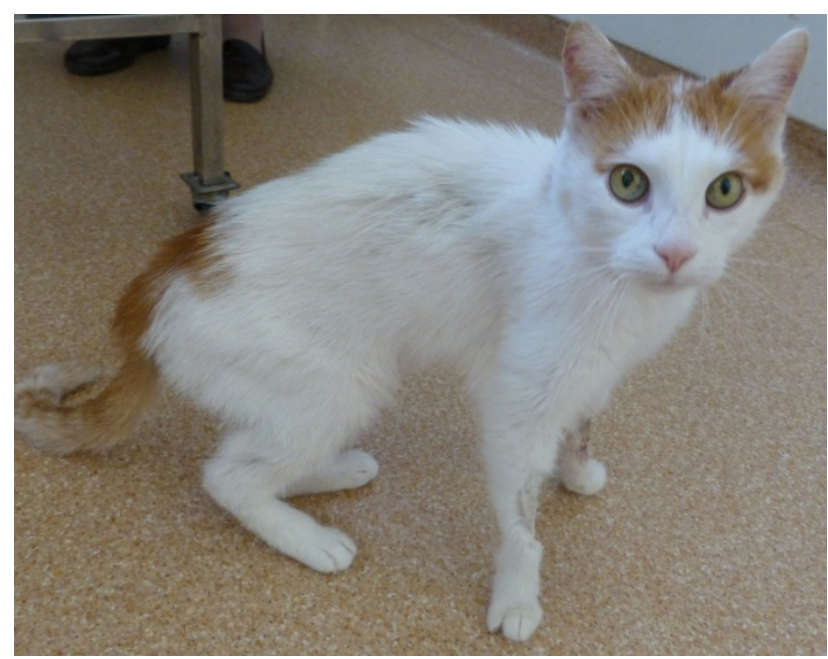

Fig. 2. Transitory hypeglycemia secondary to hyperthyroidism in a 14 year old DSH male;

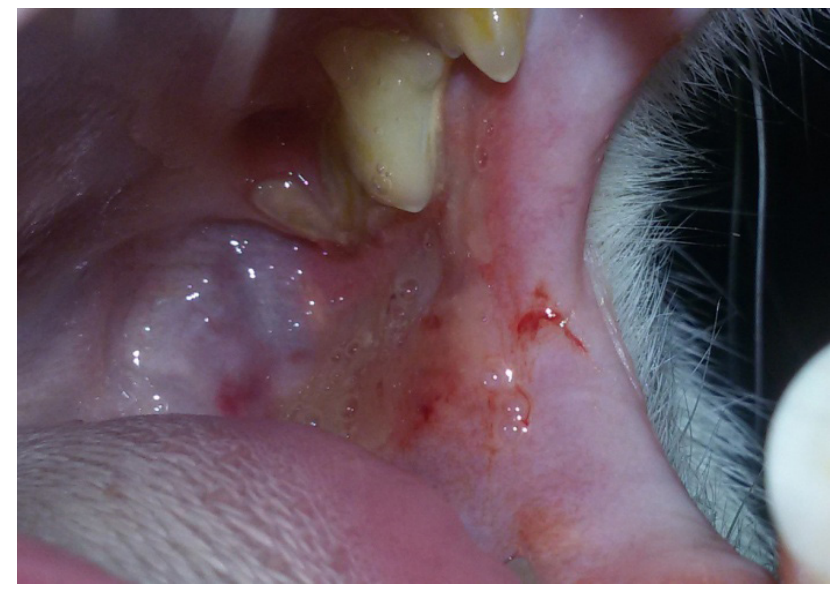

Fig. 4. DM with oscillatory insulin requirements in a 14 year old male cat with stage IV CKD. 
established diagnosis, as follows: a) primary diabetes mellitus - 27 cats, b.1) diabetes mellitus induced by hypersomathotropism - 2 cats, b.2) hyperthyroidism-1 cat, b.3) hyperadrenocorticism - 0, b.4) diabetogenic medication - 38 cats, c.1) diabetes mellitus in association with chronic kidney failure - 18 cats, c.2) heart failure - 10 cats , d) pancreatitis - 26 cats. As observed, only $41.5 \%$ of the total number of evaluated cases had DM as a primary and sole pathology. The higher proportion of $58.5 \%$ were confirmed to have other diseases, some being aggravated or even induced by DM and some being the promoters of sustained hyperglycaemia.

Diabetes mellitus, a frequent endocrinopathy affecting felines, can occur not only as a sole disease, but can also be induced by other endocrinopathies and systemic illnesses. In most cases, DM is the first condition to be diagnosed, while an insulin resistance inducing affection is suspected only after glycemic control fails (Rossmeisl et al., 2000, Blois et al., 2010). When misdiagnosed, most cases of secondary DM display difficult control of blood glucose level, highly variable insulin requirements and a shorter survival rate of cats. Also, the administration of substances meant for other complications or diseases than those present greatly reduce the cat's chances of recovery or may even hasten its exitus. Thus accurate diagnosis might be most important aspect for the future outcome of the disease. Early detection of underling diseases and administration of a proper treatment increase the chances of survival, reduce treatment costs, and provides a favourable prognosis and a better quality of life. Unfortunately, none of the tests currently used to confirm endocrine diseases hold $100 \%$ specificity. However, trough a well established differential diagnosis system and a complete panel of clinical paraclinical investigations, the rate of misdiagnosis and overlooking of other associated diseases can be highly reduce.

\section{CONCLUSION}

Diabetes mellitus diagnosis is facile and does not require advanced or expensive equipment. Unfortunately, in some cases, clinicians stop at diabetes mellitus diagnosis, overlooking important diseases which can be associated or even inducing of sustained hyperglycemia. Differential diagnosis needs to be concentrated in a logic manner and access all knowledge and rationalization of clinical experts. Paraclinical tests need to be adapted according to observed clinical signs, factors and diseases suspected to interfere with blood glucose homeostasis. An accurate diagnosis can be established only after completion of a set of logic rules and data decision, which take into consideration all present and absent clinical signs. Partial diagnosing or misdiagnosing most often lead to failure of cat recuperation and to a poorer prognosis and life expectancy.

\section{REFERENCES}

1. Blois SL, Dickie EL, Kruth SA, Allen DG (2010). Multiple endocrine diseases in cats: 15 cases (1997-2008). J Feline Med Surg 12(8): 637-642.

2. Ettinger SJ, Feldman EC (2005). Diabetes Mellitus. In: Textbook of Veterinary Internal Medicine, Elsevier Saunders, 1563-1591.

3. Gunn-Moore D (2005). Feline endocrinopathies. Vet Clin North Am Small Anim Pract 35(1): 171-210.

4. Mooney CT, Peterson ME (2004). Manual of Canine and Feline Endocrinology. $3^{\text {rd }}$ ed. British Small Animal Veterinary Association.

5. Mooney CT, Peterson M.E (2012). Manual of Canine and Feline Endocrinology, $4^{\text {th }}$ ed. British Small Animal Veterinary Association.

6. Peterson ME, Melian C, Nichols R (2001). Measurement of serum concentrations of free thyroxine, total thyroxine, and total triiodothyronine in cats with hyperthyroidism and cats with nonthyroidal disease. J Am Vet Med Assoc 218(4):529-536.

7. Rand JS (2013). Pathogenesis of feline diabetes. Vet Clin North Am Small Anim Pract 43(2): 221-231.

8. Reusch C (2011). Feline diabetes mellitus. Veterinary Focus 21(1): 9-16.

9. Rossmeisl JH, Scott-Moncrieff JC, Siems J, Snyder PW, Wells A, Anothayanontha L, Oliver JW (2000). Hyperadrenocorticism and hyperprogesteronemia in a cat with an adrenocortical adenocarcinoma. J Am Anim Hosp Assoc 36(6):512-517.

10. Rucinsky R, Cook A, Haley S, Nelson R, Zoran DL, Poundstone MA (2010). AAHA diabetes management guidelines. J Am Anim Hosp Assoc 46(3):215-224.

11. Scott-Moncrieff JC (2010). Insulin resistance in cats. Vet Clin North Am Small Anim Pract 40(2):241-257.

12. Williams TL, Peak KJ, Brodbelt D, Elliott J, Syme HM (2010). Survival and the development of azotemia after treatment of hyperthyroid cats. J Vet Intern Med 24(4): 863- 869.

13. Zini E,. Benali S, Coppola L, Guscetti F, Ackermann M, Lutz TA, Reusch CE, Aresu L (2014). Renal Morphology in Cats With Diabetes Mellitus. Vet Pathol. Vet Pathol 51(6):1143-50. 